\title{
openheart Mitral valve and left ventricular features in malignant mitral valve prolapse
}

\author{
Madalina Garbi, ${ }^{1}$ Patrizio Lancellotti, ${ }^{2,3}$ Mary N Sheppard ${ }^{4}$
}

To cite: Garbi M, Lancellotti P, Sheppard MN. Mitral valve and left ventricular features in malignant mitral valve prolapse. Open Heart 2018;5:e000925. doi:10.1136/ openhrt-2018-000925

Received 24 August 2018 Revised 4 September 2018 Accepted 26 September 2018

(A) Check for updates

(c) Author(s) (or their employer(s)) 2018. Re-use permitted under CC BY-NC. No commercial re-use. See rights and permissions. Published by BMJ.

${ }^{1}$ King's Health Partners, King's College Hospital NHS Foundation Trust, London, UK

2Department of Cardiology, GIGA Cardiovascular Sciences, University of Liège Hospital, Liège, Belgium

${ }^{3}$ Anthea Hospital, Gruppo Villa Maria Care and Research, Bari, Italy

${ }^{4}$ Cardiovascular Pathology Unit, St Georges Hospital Medical School, London, UK

\section{Correspondence to} Dr Madalina Garbi; madalina. garbi@nhs.net

\section{ABSTRACT}

Objective Mitral valve prolapse is a benign condition, however with occasional reports of sudden cardiac death or out-of-hospital cardiac arrest in the absence of severe mitral regurgitation or coronary artery disease, suggesting the existence of a malignant form. The objective of our study was to contribute to the characterisation of malignant mitral valve prolapse.

Methods We performed a retrospective analysis of pathology findings in 68 consecutive cases of sudden cardiac death with mitral valve prolapse as lone abnormal finding, reported as cause of death.

Results All mitral valve prolapse sudden death cases had mitral valve characteristics of Barlow disease, with extensive bileaflet multisegmental prolapse and dilatation of the annulus. The majority of cases (80.9\%) had microscopic left ventricular fibrosis with associated hypertrophy and degenerative features of the myocytes, and some cases (10.9\%) had right ventricular fibrosis as well.

Conclusions Malignant mitral valve prolapse is Barlow disease. Sudden cardiac death in mitral valve prolapse is due to Barlow disease, which besides the typical mitral valve degeneration may comprise a distinct Barlow disease cardiomyopathy, as suggested by myocyte degeneration and bi-ventricular involvement.

\section{INTRODUCTION}

Mitral valve prolapse (MVP) is usually a benign condition, however with sporadic reports of sudden cardiac death (SCD) or out-of-hospital cardiac arrest, often in the absence of significant mitral regurgitation, ${ }^{1-4}$ suggesting the existence of a malignant form. MVP-related SCD has low incidence; however, the risk is twice as high as for the general population. ${ }^{5}$ The characterisation of a high-risk population in need of implantable cardioverter defibrillator (ICD) for primary prevention is difficult because SCD can occur in cases with no previous clinical diagnosis. A study of out-of-hospital cardiac arrest survivors found bileaflet involvement in malignant MVP $^{6}$ and these patients had ICD-treated ventricular fibrillation recurrences.

Unexplained left ventricular systolic dysfunction, in the absence of severe mitral regurgitation or coronary artery disease, is reported occasionally ${ }^{78}$ and it was found to

\section{Key questions}

What is already known about this subject?

- Mitral valve prolapse is a benign condition, however with occasional reports of sudden cardiac death or out-of-hospital cardiac arrest in the absence of severe mitral regurgitation or coronary artery disease, suggesting the existence of a malignant form.

- Sudden cardiac death is rare in mitral valve prolapse; however, the risk is twice as high as for the general population.

- Characterisation of malignant mitral valve prolapse is needed to underpin the selection of patients in need of implantable cardioverter defibrillator for primary prevention.

What does this study add?

- Our study demonstrates association of mitral valve prolapse-related sudden cardiac death with Barlow disease rather than fibroelastic deficiency or degenerative mitral valve disease in general.

- The association was independent of mitral regurgitation severity, however related with the existence of left ventricular interstitial fibrosis and degeneration of myocytes suggestive of a distinct cardiomyopathy.

How might this impact on clinical practice?

- While further evidence is needed to underpin the selection of patients in need of implantable cardioverter defibrillator for primary prevention, our results narrow down the spectrum of high-risk characteristics to Barlow disease with associated cardiomyopathy.

- Additionally, our findings could guide further studies of myocardial fibrosis with cardiac magnetic resonance, combining T1-mapping and late gadolinium enhancement for sudden death risk stratification in mitral valve prolapse.

be an independent mortality risk factor in a community study of outcome of MVP. ${ }^{9}$

Our study aim was to contribute to the characterisation of malignant MVP by reviewing the mitral valve and left ventricular pathology findings in MVP-related SCD.

\section{METHODS}

We performed a retrospective analysis of pathology findings in 68 consecutive SCD cases with MVP reported as cause of 


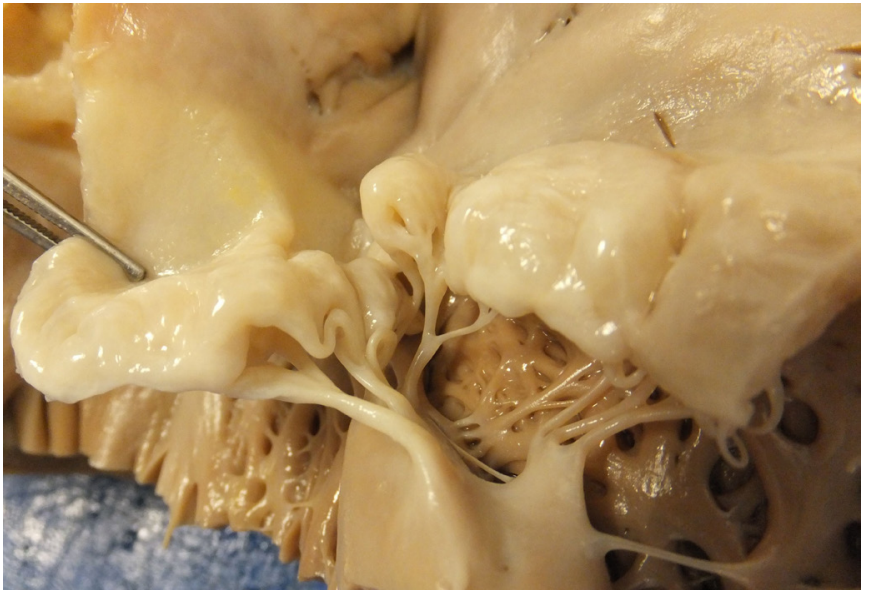

Figure 1 Barlow mitral valve with thickening and ballooning of the leaflets in between the chords and thickening and elongation of the chords.

death. The pathology assessment of all cases had been performed for diagnostic purposes, as part of a clinical autopsy, with no preconceived research hypothesis, by the same expert cardiovascular pathologist (MNS), at the Cardiovascular Pathology Reference Centre of the St George's Medical School in London. The assessment had next-of-kin consent and followed cardiac pathology guidelines ${ }^{10}$ and the methodology described by MNS. ${ }^{11}$

\section{RESULTS}

Almost half of the SCD cases $(48.5 \%)$ were younger than 35 years old, with a mean age of 38 years $(\mathrm{SD} \pm 15)$. There was marginal male predominance $(51.7 \%)$.

Clinical information was available in 50 cases $(73.5 \%)$, revealing lack of symptoms $(n=30)$ and documented mild only mitral regurgitation $(n=20)$. Six cases had relevant family history, comprising MVP with female predominance and SCD in three female relatives.

All 68 SCD cases had no evidence of coronary artery disease at autopsy.

In all cases, the mitral valve had extensive bileaflet multisegmental prolapse and dilatation of the annulus. The leaflets had pronounced permanent displacement into the left atrium (atrialisation) and thickening and ballooning in between the chords (figure 1). In all cases, there was severe tissue redundancy of the leaflets, with grade III prolapse. ${ }^{5}$ The chords were thickened and elongated, but there was no chordal rupture. In conclusion, all cases had typical mitral valve characteristics of Barlow disease, suggesting SCD association with Barlow disease specifically, rather than with fibroelastic deficiency and rather than with degenerative mitral valve disease or MVP in general.

There was no associated tricuspid valve prolapse; the tricuspid valve was normal in all cases.

In only 17 cases $(25 \%)$, there was hypertrophy with increased heart weight $(>500 \mathrm{~g})$ and left ventricular wall thickness $(>15 \mathrm{~mm})$.

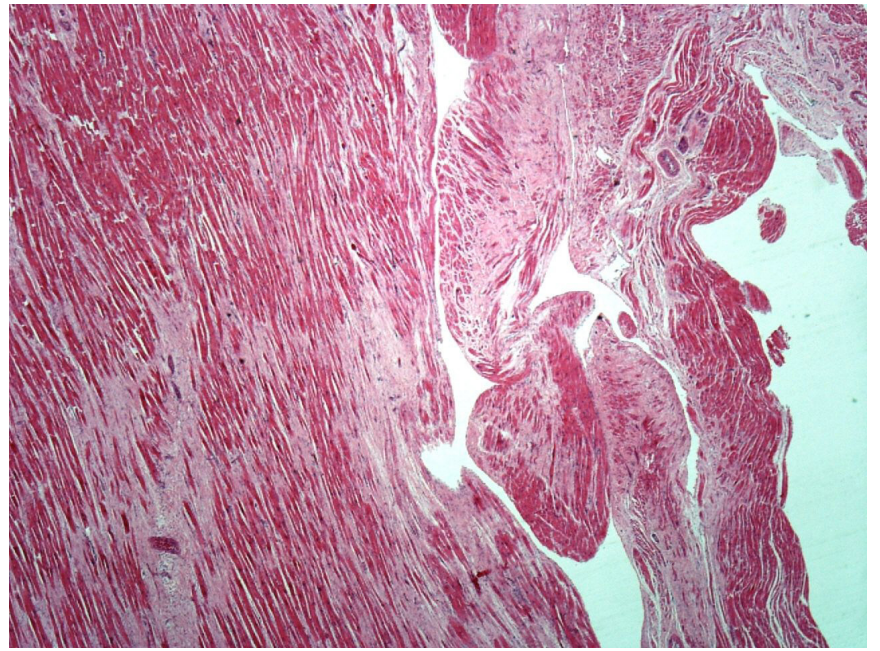

Figure 2 Ventricular fibrosis in Barlow disease.

The existence of MVP without chordal rupture prompted thorough assessment for myocardial fibrosis in all cases; this is because, in the absence of chordal rupture, based on pathology guidelines, ${ }^{10} \mathrm{MVP}$ is a highly probable cause of the SCD only in the presence of associated myocardial fibrosis. Macroscopic analysis found no evidence of ventricular wall fibrosis in any of the 68 cases. However, microscopic analysis found ventricular fibrosis in the majority of cases $(n=55,80.9 \%)$, confined to the left ventricle in 49 cases $(89.1 \%)$ and involving the right ventricle as well in six cases $(10.9 \%)$. The fibrosis (figure 2) was focal, usually fine and interstitial within the inner wall, involving particularly the inner subendocardium, trabeculae and papillary muscles. In the left ventricle, within the inner third of the basal posterior wall, the fibrosis became confluent, with extensive replacement fibrosis extending into the trabeculae and posteromedial papillary muscle. The fibrosis was never epicardial or diffuse throughout the wall. Because MVP with associated fibrosis is a highly probable but not certain cause of SCD based on pathology guidelines, histological analysis of the myocardium was also performed. Associated with the myocardial fibrosis, there was hypertrophy and degenerative features of the myocytes with focal cytoplasmic vacuolation (figure 3) suggestive of existence of a cardiomyopathy. There was no myocyte disarray to establish a diagnosis of hypertrophic cardiomyopathy, and there was no associated fatty infiltration to establish a diagnosis of arrhythmogenic cardiomyopathy. All cases had posterior left ventricular wall and posterior papillary muscle involvement. Twelve cases had lateral and anterior left ventricular wall involvement as well. Sixteen cases had midwall interventricular septum fibrosis, which became confluent with the basal posterior fibrosis. In the right ventricle, the fibrosis was usually subendocardial, with fine interstitial pattern in the posterior wall extending into the basal posterior left ventricular fibrosis. There was interventricular septum fibrosis in all cases with right ventricular involvement. 




Figure 3 Degenerated myocytes with cytoplasmic vacuolation but no disarray in Barlow disease.

The SCD cases with ventricular fibrosis had marked dilatation of the mitral valve annulus in the absence of left ventricular cavity dilatation, suggesting more advanced mitral valve degeneration, not necessarily associated with more severe mitral regurgitation, as $29 \%$ of cases had documented mild only mitral regurgitation.

\section{DISCUSSION}

We report, to the best of our knowledge, the largest number of SCD cases with MVP as lone abnormal finding, having had consistent systematic pathology analysis performed for diagnostic purposes by the same expert cardiovascular pathologist (MNS), guided by no preconceived research hypothesis. The reported findings were only retrospectively reviewed and interpreted by the authors of this paper with the aim to characterise malignant MVP. Our study found Barlow disease mitral valve characteristics and features of a distinct cardiomyopathy in MVP-related SCD.

Association of MVP with a cardiomyopathy ${ }^{7}$ was hypothesised soon after the description by Barlow of late systolic mitral regurgitation and 'aneurysmal' posterior leaflet protrusion within the left atrium ${ }^{12}$ in patients with systolic click. This hypothesis was based on the typical 'ballerina foot' angiographic systolic appearance of the contrast-delineated left ventricular cavity in MVP, interpreted as being due to an abnormal myocardial contraction pattern. ${ }^{13}$

Non-invasive cardiac imaging findings enhanced suspicion of potential association of MVP with a cardiomyopathy awaiting to be confirmed. ${ }^{814}$ Myocardial fibrosis on cardiac magnetic resonance was found to be associated with reduced global longitudinal strain and exercise capacity ${ }^{8}$ but not with the severity of mitral regurgitation in $\mathrm{MVP}^{14}$; therefore, a coexistent cardiomyopathy could explain the myocardial fibrosis. Furthermore, myocardial fibrosis diagnosed on cardiac magnetic resonance, particularly papillary muscle fibrosis,${ }^{15}$ was found to be associated with potentially malignant complex ventricular arrhythmia ${ }^{14}{ }^{15}$; therefore, a coexistent cardiomyopathy could explain malignant MVP. A higher incidence of arrhythmia at rest or on exertion was reported in SCD or out-of-hospital cardiac arrest cases due to malignant MVP. $^{261617}$

Electrocardiographic QT dispersion in MVP was found to be associated with the echocardiographic degree of MVP and anterior leaflet thickness ${ }^{18}$; however, it is also widely used as SCD risk marker in cardiomyopathy. This reported association of QT dispersion with more advanced mitral valve degeneration ${ }^{18}$ parallels our finding of association of myocardial fibrosis with more advanced mitral valve degeneration in SCD. Similarly, MVP-related SCD association with more advanced mitral valve degeneration characterised by greater annulus circumference and greater length and thickness of leaflets has been previously reported by the group of Farb. ${ }^{1}$ The same group reported as well the existence of endocardial plaque in MVP-related SCD, ${ }^{1}$ although their study focused on the mitral valve morphology and not on the left ventricle.

SCD in degenerative mitral valve disease with severe mitral regurgitation ${ }^{16}$ can be easily explained as due to heart failure ${ }^{19}$; this is the case in a large published study of flail mitral valve leaflet, ${ }^{16}$ likely due to fibroelastic deficiency. ${ }^{20}$ On the contrary, SCD in degenerative mitral valve disease with no chordal rupture and no significant mitral regurgitation ${ }^{2-46}$ is more difficult to explain; this is the case in the published study of malignant bileaflet MVP, ${ }^{6}$ likely due to Barlow disease. ${ }^{20}$ Detailed characterisation of malignant MVP is needed to inform SCD risk stratification in MVP and select patients in need of ICD implantation for primary prevention.

Our study demonstrates MVP-related SCD association with Barlow disease, regardless of mitral regurgitation severity, more likely with left ventricular interstitial fibrosis and degeneration of myocytes suggestive of a distinct cardiomyopathy. These findings narrow down the spectrum of MVP-related SCD high-risk characteristics to Barlow disease with associated cardiomyopathy.

Papillary muscle traction in systole was described in Barlow disease, ${ }^{21}$ consequence of pronounced MVP, and this was thought to be arrhythmogenic and related with SCD. The posterior leaflet middle scallop is larger and more likely and more prominently prolapsing in all forms of MVP, including Barlow disease. The papillary muscle traction and the predominant posterior leaflet involvement may explain the posterior wall and posterior papillary muscle fibrosis in all our cases. The fibrosis found at postmortem or on late gadolinium enhancement cardiac magnetic resonance was interpreted as due to 'myocardial stretch by the prolapsing leaflet' in a recent study of MVP-related SCD.$^{22}$ However, the existence of ventricular fibrosis in areas not affected by traction or 'stretch' and, particularly, the existence of ventricular fibrosis within the right ventricle in our study, together with the coexistent hypertrophy and degeneration of myocytes, suggest the existence of a cardiomyopathy. 
Our study found myocardial fibrosis on microscopic and not macroscopic pathology analysis. The fibrosis was extensive but focal rather than diffuse, always involving the posterior papillary muscle. Cardiac magnetic resonance T1 mapping is thought to detect microscopic or diffuse fibrosis, while late gadolinium enhancement detects patchy macroscopic fibrosis. This may explain differences in prevalence of myocardial fibrosis detected with cardiac magnetic resonance in patients with complex ventricular arrhythmia ${ }^{14}$ compared with our study. Diffuse myocardial fibrosis on T1 mapping was found in $91 \%$ of cases, with only $41 \%$ having localised papillary muscle fibrosis on late gadolinium enhancement. ${ }^{14}$ Our findings could guide further studies of myocardial fibrosis with cardiac magnetic resonance, combining T1 mapping and late gadolinium enhancement for SCD risk stratification in MVP.

Our study had incomplete clinical data to corroborate with pathology findings, which is a limitation common for SCD pathology studies, due to the lack of previous diagnosis of disease in many SCD cases. Correlation of pathology and imaging findings would have been desirable; however, in cases having had an echocardiogram prior to $\mathrm{SCD}$, the echocardiographic images were not available to the investigators for review.

Being based on pathology findings at autopsy performed for diagnostic rather than research purposes, our study does not include assessment for existence of mitral annular disjunction, anatomical variation present in MVP and also in the general population. ${ }^{23}{ }^{24}$ The incidence of mitral annular disjunction is very high in MVP, and consequently also in MVP-related SCD or arrhythmia, having no use for SCD risk stratification. A recent pathology study ${ }^{25}$ reports mitral annular disjunction in MVP SCD cases but also in controls without MVP, differing though in measured length.

\section{CONCLUSION}

Malignant MVP is Barlow disease. MVP-related SCD is due to Barlow disease, which besides the typical mitral valve degeneration may comprise a distinct cardiomyopathy. While further evidence is needed to underpin the selection of patients in need of ICD for primary prevention, our results narrow down the spectrum of MVP-related SCD high-risk characteristics to Barlow disease with associated cardiomyopathy.

Contributors MG has contributed to planning, conduct, reporting of the work described in the article and is responsible for the overall content as guarantor. PL has contributed to the planning, conduct and reporting of the work described in the article. MNS contributed to planning, conduct, reporting of the work described in the article and is responsible for the overall content as guarantor.

Funding The authors have not declared a specific grant for this research from any funding agency in the public, commercial or not-for-profit sectors.

Competing interests None declared.

Patient consent Not required.

Provenance and peer review Not commissioned; internally peer reviewed.
Data sharing statement № additional unpublished data from the study are available.

Open access This is an open access article distributed in accordance with the Creative Commons Attribution Non Commercial (CC BY-NC 4.0) license, which permits others to distribute, remix, adapt, build upon this work non-commercially, and license their derivative works on different terms, provided the original work is properly cited, appropriate credit is given, any changes made indicated, and the use is non-commercial. See: http://creativecommons.org/licenses/by-nc/4.0/

\section{REFERENCES}

1. Farb A, Tang AL, Atkinson JB, et al. Comparison of cardiac findings in patients with mitral valve prolapse who die suddenly to those who have congestive heart failure from mitral regurgitation and to those with fatal noncardiac conditions. Am J Cardiol 1992;70:234-9.

2. Anders S, Said S, Schulz F, et al. Mitral valve prolapse syndrome as cause of sudden death in young adults. Forensic Sci Int 2007;171(23):127-30.

3. Dollar AL, Roberts WC. Morphologic comparison of patients with mitral valve prolapse who died suddenly with patients who died from severe valvular dysfunction or other conditions. J Am Coll Cardiol 1991;17:921-31.

4. Chesler E, King RA, Edwards JE. The myxomatous mitral valve and sudden death. Circulation 1983;67:632-9.

5. Kligfield P, Levy D, Devereux RB, et al. Arrhythmias and sudden death in mitral valve prolapse. Am Heart J 1987;113:1298-307.

6. Sriram CS, Syed FF, Ferguson ME, et al. Malignant bileaflet mitral valve prolapse syndrome in patients with otherwise idiopathic outof-hospital cardiac arrest. J Am Coll Cardiol 2013;62:222-30.

7. Gulotta SJ, Gulco L, Padmanabhan V, et al. The syndrome of systolic click, murmur, and mitral valve prolapse-a cardiomyopathy? Circulation 1974;49:717-28.

8. Edwards NC, Moody WE, Yuan M, et al. Quantification of left ventricular interstitial fibrosis in asymptomatic chronic primary degenerative mitral regurgitation. Circ Cardiovasc Imaging 2014;7:946-53.

9. Avierinos JF, Gersh BJ, Melton LJ, et al. Natural history of asymptomatic mitral valve prolapse in the community. Circulation 2002;106:1355-61.

10. Basso C, Burke M, Fornes P. Association for European Cardiovascular Pathology. Guidelines for autopsy investigation of sudden cardiac death. Virchows Arch 2008;452:11-18.

11. Sheppard MN. Approach to the cardiac autopsy. J Clin Pathol 2012;65:484-95.

12. Barlow JB, Bosman CK. Aneurysmal protrusion of the posterior leaflet of the mitral valve. Am Heart $J$ 1966;71:166-78.

13. Scampardonis G, Yang SS, Maranhão V, et al. Left ventricular abnormalities in prolapsed mitral leaflet syndrome. Review of eightyseven cases. Circulation 1973;48:287-97.

14. Bui AH, Roujol S, Foppa M, et al. Cardiac magnetic resonance evidence of diffuse myocardial fibrosis in patients with mitral valve prolapse. J Cardiovasc Magn Reson 2015;17(Suppl 1):337):P337.

15. Han Y, Peters DC, Salton CJ, et al. Cardiovascular magnetic resonance characterization of mitral valve prolapse. JACC Cardiovasc Imaging 2008;1:294-303.

16. Grigioni F, Enriquez-Sarano M, Ling LH, et al. Sudden death in mitral regurgitation due to flail leaflet. J Am Coll Cardiol 1999;34:2078-85.

17. Babuty D, Fauchier L, Neel C, et al. Arrhythmias and sudden death in patients with mitral valve prolapse. Card Electrophysiol Rev 1997;1(1/2):264-7.

18. Zouridakis EG, Parthenakis FI, Kochiadakis GE, et al. QT dispersion in patients with mitral valve prolapse is related to the echocardiographic degree of the prolapse and mitral leaflet thickness. Europace 2001;3:292-8.

19. Carabello BA. Sudden death in mitral regurgitation: why was I so surprised? J Am Coll Cardiol 1999;34:2086-7.

20. Anyanwu AC, Adams DH. Etiologic classification of degenerative mitral valve disease: Barlow's disease and fibroelastic deficiency. Semin Thorac Cardiovasc Surg 2007;19:90-6.

21. Sanfilippo AJ, Harrigan P, Popovic AD, et al. Papillary muscle traction in mitral valve prolapse: quantitation by two-dimensional echocardiography. J Am Coll Cardiol 1992;19:564-71.

22. Basso C, Perazzolo Marra M, Rizzo S, et al. Arrhythmic mitral valve prolapse and sudden cardiac death. Circulation 2015;132:556-66.

23. Hutchins GM, Moore GW, Skoog DK. The association of floppy mitral valve with disjunction of the mitral annulus fibrosus. $N$ Engl $J$ Med 1986;314:535-40. 
24. Lancellotti P, Garbi M. Malignant mitral valve prolapse: substrates to ventricular remodeling and arrhythmias. Circ Cardiovasc Imaging 2016;9:e005248.
25. Perazzolo Marra M, Basso C, De Lazzari M, et al. Morphofunctional abnormalities of mitral annulus and arrhythmic mitral valve prolapse. Circ Cardiovasc Imaging 2016;9:e005030. 